\title{
Electronic Communication in Luminescent Dicyanorhenate-Bridged Homotrinuclear Rhenium(I) Complexes.
}

Yelan Xiao, ${ }^{a}$ Apple Wai-Yi Cheung, ${ }^{a}$ Sze-Wing Lai, ${ }^{a}$ Shun-Cheung Cheng, ${ }^{\text {a }}$ Shek-Man Yiu, ${ }^{a}$ Chi-Fai Leung, ${ }^{\mathrm{b}}$ Chi-Chiu $\mathrm{Ko}^{\mathrm{a}, *}$

${ }^{a}$ Department of Chemistry, City University of Hong Kong, Tat Chee Avenue, Kowloon, Hong Kong, China.

${ }^{b}$ Department of Science and Environmental Studies, The Education University of Hong Kong, 10 Lo Ping Road, Tai Po, N. T. Hong Kong, China.

E-mail: vinccko@cityu.edu.hk

Supporting Information 
Physical Measurements and Instrumentation. ${ }^{1} \mathrm{H}$ NMR spectra were recorded on a Bruker AV400 (400 MHz) FT-NMR spectrometer. Chemical shifts $(\delta, \mathrm{ppm})$ were reported relative to tetramethylsilane $\left(\mathrm{Me}_{4} \mathrm{Si}\right)$. All positive-ion ESI mass spectra were recorded on a Perkin-Elmer Sciex API 365 mass spectrometer. Elemental analyses were performed on an Elementar Vario EL III Analyzer. Electronic absorption spectra were recorded on a Hewlett-Packard 8452A diode array spectrophotometer. Steady state emission and excitation spectra at room temperature and at $77 \mathrm{~K}$ were recorded on a Horiba Jobin Yvon Fluorolog-3-TCSPC spectrofluorometer. Solutions were rigorously degassed on a high-vacuum line in a two-compartment cell with no less than four successive freeze pump-thaw cycles. Measurements of the $\mathrm{EtOH}-\mathrm{MeOH}(4: 1 \mathrm{v} / \mathrm{v})$ glass samples at $77 \mathrm{~K}$ were carried out with the dilute $\mathrm{EtOH}-\mathrm{MeOH}$ sample solutions contained in a quartz tube inside a liquid nitrogen-filled quartz optical Dewar flask. Luminescence quantum yields were determined using the optically dilution method described by Demas and Crosby ${ }^{1}$ with an aqueous solution of $\left[\mathrm{Ru}(\mathrm{bpy})_{3}\right] \mathrm{Cl}_{2}\left(\phi_{\mathrm{em}}=0.063^{2}\right.$ with $436-\mathrm{nm}$ excitation) as the refer ence. Luminescence lifetimes of the samples were measured using time-correlated single photon counting (TCSPC) technique on the TCSPC spectrofluorometer in a Fast MCS mode with a NanoLED-375LH excitation source, which has its excitation peak wavelength at $375 \mathrm{~nm}$ and pulse width shorter than 750 ps. The photon counting data were analyzed by Horiba Jobin Yvon Decay Analysis Software.

Cyclic voltammetric measurements were performed by using a $\mathrm{CH}$ Instruments, Inc. Model CHI 620 Electrochemical Analyzer. Electrochemical measurements were performed in acetonitrile solutions with $0.1 \mathrm{M}^{n} \mathrm{Bu}_{4} \mathrm{NPF}_{6}$ as the supporting electrolyte at room temperature. The reference electrode was a $\mathrm{Ag} / \mathrm{AgNO}_{3}(0.1 \mathrm{M}$ in acetonitrile) electrode, and the working electrode was a glassy carbon electrode (1 mm diameter) with a platinum wire as the auxiliary electrode. The working electrode surface was polished with a $1 \mu \mathrm{m} \alpha$-alumina slurry (Linde) and then a $0.3 \mu \mathrm{m} \quad \alpha$-alumina slurry (Linde) on a microcloth (Buehler Co.). The ferrocenium/ferrocene couple $\left(\mathrm{FeCp}_{2}{ }^{+/ 0}\right)$ was used as the internal reference. All solutions for electrochemical studies were deaerated with pre-purified argon gas prior to measurements.

Controlled-potential coulometry was performed by electrolysis in a two-compartment cell using a CH Instrument Inc. CHI 620 Electrochemical Analyser. The anodic compartment containing $0.5 \mathrm{mM}$ of the complex, the glassy carbon plate working electrode $(1.5 \times 1 \mathrm{~cm})$ and the saturated calomel reference electrode in $0.1 \mathrm{M}^{n} \mathrm{Bu}_{4} \mathrm{NPF}_{6}$ acetonitrile solution $(10 \mathrm{~mL})$ was separated from the cathodic compartment containing a platinum-grid counter electrode in $0.4 \mathrm{M}$ 
${ }^{n} \mathrm{Bu}_{4} \mathrm{NClO}_{4}$ acetonitrile solution by a glass frit. The potential for the electrolysis was first determined by the cyclic voltammetry of the complex solution in the same electrolysis cell. The electrical charge (Q) in coulomb required for respective oxidation process was obtained by coulometry at the electrolysis potential until the current becomes steady. The number of equivalent charge $(n)$ in Faraday $(\mathrm{F}=96487 \mathrm{C})$ for the reaction of 1 mole was estimated by the equation $\mathrm{Q}=n \mathrm{~F}(\mathrm{~m} / \mathrm{M})$, where $\mathrm{m}$ and $\mathrm{M}$ are the mass and molecular weight of the complex.

X-Ray Crystal Structure Determination. The crystal structures were determined on an Oxford Diffraction Gemini S Ultra X-ray single crystal diffractometer using graphite monochromatized $\mathrm{Cu}-\mathrm{K} \alpha(\lambda=1.54184 \AA)$. The structure was solved by direct methods employing SHELXL-97 program $^{3}$ on PC. Re and many non-H atoms were located according to the direct methods. The positions of other non-hydrogen atoms were found after successful refinement by full-matrix least-squares using SHELXL-97 program on PC. In the final stage of least-squares refinement, all non-hydrogen atoms were refined anisotropically. $\mathrm{H}$ atoms were generated by program SHELXL-97. The positions of $\mathrm{H}$ atoms were calculated based on riding mode with thermal parameters equal to 1.2 times that of the associated $\mathrm{C}$ atoms, and participated in the calculation of final R-indices.

Computational Details. All calculations were carried out using the Gaussian 09, version B.01. ${ }^{4}$ The structures of the ground state and of the complexes 2, 5 and $\mathbf{6}$ were optimized using Density Functional Theory (DFT) with the B3LYP hybrid functional. ${ }^{5}$ A combination of 6-31+G(d) (for elements H, C, N, O and P) and LANL2DZ ${ }^{6}$ (for Re) basis sets was employed. Vibrational analysis was performed to ensure that all optimized structures are located at potential energy minima. The effect of the solvent was taken account by the polarized continuum model with integral equation formalism (IEF-PCM). ${ }^{7}$ 
Table S1. Crystal and structure determination data for $\mathbf{2}-\mathbf{6}$.

\begin{tabular}{|c|c|c|c|c|c|}
\hline & 2 & 3 & 4 & 5 & 6 \\
\hline Formula & $\begin{array}{l}\mathrm{C}_{80} \mathrm{H}_{54} \mathrm{~N}_{8} \mathrm{O}_{6} \mathrm{P}_{2} \mathrm{Re}_{3} \cdot \mathrm{CF}_{3} \\
\mathrm{SO}_{3} \cdot 0.5\left(\mathrm{CH}_{2} \mathrm{Cl}_{2}\right)\end{array}$ & $\begin{array}{l}\mathrm{C}_{80} \mathrm{H}_{50} \mathrm{Br}_{4} \mathrm{~N}_{8} \mathrm{O}_{6} \mathrm{P}_{2} \mathrm{Re}_{3} \\
\cdot \mathrm{PF}_{6} \cdot 3.75\left(\mathrm{C}_{4} \mathrm{H}_{10} \mathrm{O}\right)\end{array}$ & $\begin{array}{l}\mathrm{C}_{74} \mathrm{H}_{56} \mathrm{~N}_{4} \mathrm{O}_{8} \mathrm{P}_{4} \mathrm{Re}_{3} \cdot \\
\mathrm{PF}_{6} \cdot\left(\mathrm{C}_{4} \mathrm{H}_{10} \mathrm{O}\right)\end{array}$ & $\mathrm{C}_{26} \mathrm{H}_{8} \mathrm{~N}_{4} \mathrm{O}_{12} \mathrm{Re}_{3} \cdot \mathrm{PF}_{6}$ & $\begin{array}{l}\mathrm{C}_{47} \mathrm{H}_{24} \mathrm{~N}_{8} \mathrm{O}_{8} \mathrm{Re}_{3} \cdot \mathrm{C} \\
\mathrm{F}_{3} \mathrm{SO}_{3} \cdot 0.5(\mathrm{MeCN})\end{array}$ \\
\hline$M_{r}$ & 2035.38 & 2582.38 & 2030.80 & 1271.93 & 1602.52 \\
\hline$T,[\mathrm{~K}]$ & $133(2)$ & $133(2)$ & $133(2)$ & $133(2)$ & $133(2)$ \\
\hline$a,[\AA]$ & $22.6282(8)$ & $16.6370(5)$ & $13.9922(5)$ & $12.0778(9)$ & $9.9500(4)$ \\
\hline$b,[\AA]$ & $24.7070(6)$ & $24.1112(5)$ & $14.1690(4)$ & $12.6002(8)$ & $13.6384(5)$ \\
\hline$c,[\AA]$ & $14.3599(4)$ & $25.5696(5)$ & $20.1569(5)$ & $13.2887(10)$ & $21.3878(8)$ \\
\hline$\alpha,[\operatorname{deg}]$ & 90.00 & $83.993(2)$ & $105.456(2)$ & $74.291(6)$ & 89.019 (3) \\
\hline$\beta,[\operatorname{deg}]$ & $106.657(4)$ & $82.343(2)$ & $103.084(2)$ & $66.707(7)$ & $81.600(3)$ \\
\hline$\gamma,[\operatorname{deg}]$ & 90.00 & $86.533(2)$ & $92.113(2)$ & $81.181(6)$ & $70.432(3)$ \\
\hline$V,\left[\AA^{3}\right]$ & $7691.4(4)$ & $10098.4(4)$ & $3731.54(19)$ & $1785.7(2)$ & $2703.83(18)$ \\
\hline Crystal color & Red & Red & Red & Orange & Orange \\
\hline Crystal system & Monoclinic & Triclinic & Triclinic & Triclinic & Triclinic \\
\hline Space group & $P 2_{1} / c$ & $P \overline{1}$ & $P \overline{1}$ & $P \overline{1}$ & $P \overline{1}$ \\
\hline$Z$ & 4 & 4 & 2 & 2 & 2 \\
\hline$F(000)$ & 3948 & 5022 & 1976 & 1164 & 1522 \\
\hline$D_{c,}\left[\mathrm{~g} \mathrm{~cm}^{-3}\right]$ & 1.758 & 1.699 & 1.807 & 2.366 & 1.968 \\
\hline $\begin{array}{l}\text { Crystal dimensions } \\
{[\mathrm{mm}]}\end{array}$ & $0.2 \times 0.19 \times 0.01$ & $0.72 \times 0.12 \times 0.03$ & $0.33 \times 0.12 \times 0.01$ & $0.84 \times 0.11 \times 0.02$ & $0.61 \times 0.24 \times 0.02$ \\
\hline$\lambda,[\AA]$ & 1.54184 & 1.54184 & 1.54184 & 1.5418 & 1.54184 \\
\hline$\mu / \mathrm{mm}^{-1}$ & 10.59 & 9.759 & 10.96 & 20.74 & 13.886 \\
\hline Collection range & $\begin{array}{l}3.6 \leq \theta \leq 71.7^{\circ} \\
\text { (h: }-27 \text { to } 25 ; k:-30 \text { to } \\
20 ; l:-17 \text { to } 17 \text { ) }\end{array}$ & $\begin{array}{l}3.2 \leq \theta \leq 71.8^{\circ} \\
\text { ( } h:-19 \text { to } 20 ; k:-21 \\
\text { to } 29 ; l:-30 \text { to } 31)\end{array}$ & $\begin{array}{l}3.3 \leq \theta \leq 71.7^{\circ} \\
\text { (h: }-17 \text { to } 17 ; k:- \\
17 \text { to } 14 ; l:-18 \text { to } \\
24)\end{array}$ & $\begin{array}{l}3.7 \leq \theta \leq 67.0^{\circ} \\
(h:-13 \text { to } 14 ; k:-11 \text { to } \\
15 ; l:-12 \text { to } 15)\end{array}$ & $\begin{array}{l}3.4 \leq \theta \leq 66.9^{\circ} \\
\text { (h:-11 to } 10 ; k:- \\
16 \text { to } 11 ; l:-24 \text { to } \\
25 \text { ) }\end{array}$ \\
\hline $\begin{array}{l}\text { Completeness to } \\
\text { theta }\end{array}$ & $96.7 \%$ & 0.974 & 0.974 & 0.993 & $99.4 \%$ \\
\hline No. of data collected & 31682 & 78623 & 27985 & 11915 & 19228 \\
\hline No. of unique data & 14742 & 38602 & 14229 & 6318 & 9573 \\
\hline $\begin{array}{l}\text { No. of data used in } \\
\text { refinement, } m\end{array}$ & 12195 & 33261 & 13462 & 5539 & 9304 \\
\hline $\begin{array}{l}\text { No. of parameters } \\
\text { refined, } p\end{array}$ & 1018 & 2182 & 937 & 469 & 733 \\
\hline$R^{a}$ & 0.0478 & 0.0615 & 0.0309 & 0.0636 & 0.0373 \\
\hline$w R^{a}$ & 0.1427 & 0.1741 & 0.0848 & 0.19880 & 0.1089 \\
\hline Goodness-of-fit, $S$ & 1.09 & 1.044 & 1.080 & 1.119 & 1.08 \\
\hline $\begin{array}{l}\text { Maximum shift, } \\
(\Delta / \sigma)_{\max }\end{array}$ & 0.002 & 0.002 & 0.002 & 0.001 & 0.001 \\
\hline $\begin{array}{l}\text { Residual extrema in } \\
\text { final difference } \\
\text { map, } \mathrm{e}^{-3}\end{array}$ & $+2.76,-1.87$ & $+4.79,-2.96$ & $+3.37,-1.84$ & $+2.92,-1.74$ & $+2.70,-1.46$ \\
\hline
\end{tabular}

\footnotetext{
${ }^{a} w=1 /\left[\sigma^{2}\left(F_{\mathrm{o}}{ }^{2}\right)+(a p)^{2}+b P\right]$, where $P=\left[2{F_{\mathrm{c}}}^{2}+\operatorname{Max}\left(F_{\mathrm{o}}^{2}, 0\right)\right] / 3$
} 
Table S2. Selected bond distances $(\AA)$ and angles $\left(^{\circ}\right)$ with estimated standard deviations (e.s.d.s.) in parentheses for $\mathbf{2}-\mathbf{6}$.

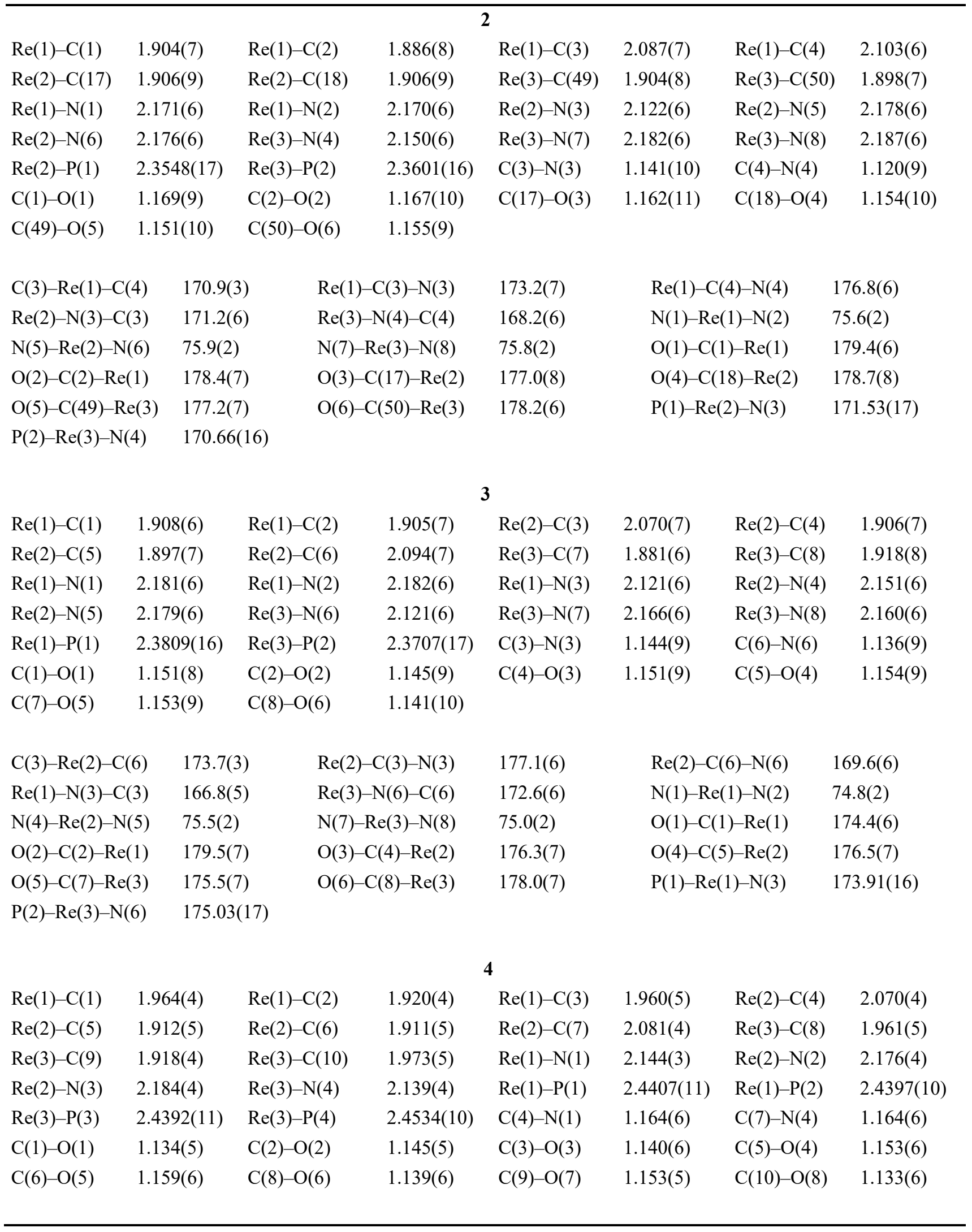




\begin{tabular}{|c|c|c|c|c|c|c|c|c|c|c|}
\hline \multicolumn{2}{|c|}{$C(4)-\operatorname{Re}(2)-C(7)$} & \multicolumn{2}{|c|}{$173.23(16)$} & \multicolumn{2}{|c|}{$\operatorname{Re}(2)-\mathrm{C}(4)-\mathrm{N}(1)$} & \multicolumn{2}{|l|}{$177.2(4)$} & \multicolumn{2}{|c|}{$\operatorname{Re}(2)-\mathrm{C}(7)-\mathrm{N}(4)$} & $177.5(4)$ \\
\hline \multicolumn{2}{|c|}{$\operatorname{Re}(1)-\mathrm{N}(1)-\mathrm{C}(4)$} & \multicolumn{2}{|c|}{$165.4(3)$} & \multicolumn{2}{|c|}{$\operatorname{Re}(3)-\mathrm{N}(4)-\mathrm{C}(7)$} & \multicolumn{2}{|l|}{$174.8(3)$} & \multicolumn{2}{|c|}{$\mathrm{C}(2)-\operatorname{Re}(1)-\mathrm{N}(1)$} & $174.83(16)$ \\
\hline \multicolumn{2}{|c|}{$\mathrm{C}(9)-\operatorname{Re}(3)-\mathrm{N}(4)$} & \multicolumn{2}{|c|}{$175.10(16)$} & \multicolumn{2}{|c|}{$\mathrm{N}(2)-\operatorname{Re}(2)-\mathrm{N}(3)$} & \multicolumn{2}{|l|}{$75.68(17)$} & \multicolumn{2}{|c|}{$\mathrm{O}(1)-\mathrm{C}(1)-\operatorname{Re}(1)$} & $176.3(4)$ \\
\hline \multicolumn{2}{|c|}{$\mathrm{O}(2)-\mathrm{C}(2)-\operatorname{Re}(1)$} & \multicolumn{2}{|c|}{$179.8(5)$} & \multicolumn{2}{|c|}{$\mathrm{O}(3)-\mathrm{C}(3)-\operatorname{Re}(1)$} & \multicolumn{2}{|l|}{$174.8(4)$} & \multicolumn{2}{|c|}{$\mathrm{O}(4)-\mathrm{C}(5)-\operatorname{Re}(2)$} & $179.3(4)$ \\
\hline \multicolumn{2}{|c|}{$\mathrm{O}(5)-\mathrm{C}(6)-\operatorname{Re}(2)$} & \multicolumn{2}{|c|}{$178.7(4)$} & $\mathrm{O}(6)$ & $C(8)-\operatorname{Re}(3)$ & 177.1(4) & & $\mathrm{O}(7)-\mathrm{C}($ & 9) $-\operatorname{Re}(3)$ & $177.9(4)$ \\
\hline $\mathrm{O}(8)-\mathrm{C}(10)-\mathrm{I}$ & (3) & 177.2 & & $\mathrm{P}(1)$ & e(1)-P(2) & $80.58(3)$ & & $\mathrm{P}(3)-\mathrm{Re}$ & (3) $-\mathrm{P}(4)$ & $81.65(3)$ \\
\hline & & & & & & 5 & & & & \\
\hline $\operatorname{Re}(1)-C(1)$ & 1.87 & $5(14)$ & $\operatorname{Re}$ & (2) & $1.907(12)$ & $\operatorname{Re}(1)-C(3$ & & $2.097(9)$ & $\operatorname{Re}(1)-C(4)$ & $2.089(12)$ \\
\hline $\operatorname{Re}(2)-C(17)$ & 2.03 & $0(12)$ & $\operatorname{Re}$ & (18) & $2.045(14)$ & $\operatorname{Re}(2)-C(1$ & & $2.011(11)$ & $\operatorname{Re}(2)-C(20$ & $2.027(14)$ \\
\hline $\operatorname{Re}(2)-C(21)$ & 1.93 & $9(11)$ & $\operatorname{Re}$ & (22) & $1.975(12)$ & $\operatorname{Re}(3)-C(2$ & & $2.009(12)$ & $\operatorname{Re}(3)-C(24$ & $2.042(13)$ \\
\hline $\operatorname{Re}(3)-C(25)$ & 2.01 & 1(12) & $\operatorname{Re}$ & (26) & $1.980(12)$ & $\operatorname{Re}(1)-\mathrm{N}(1$ & & $2.229(10)$ & $\operatorname{Re}(1)-\mathrm{N}(2)$ & $2.193(9)$ \\
\hline $\operatorname{Re}(2)-\mathrm{N}(3)$ & 2.10 & $4(8)$ & $\operatorname{Re}$ & $\mathrm{J}(4)$ & $2.108(9)$ & $\mathrm{C}(3)-\mathrm{N}(3)$ & & $1.157(15)$ & $\mathrm{C}(4)-\mathrm{N}(4)$ & $1.143(17)$ \\
\hline $\mathrm{C}(1)-\mathrm{O}(1)$ & 1.20 & $4(17)$ & $\mathrm{C}(2$ & & $1.140(15)$ & $\mathrm{C}(17)-\mathrm{O}($ & & $1.112(16)$ & $\mathrm{C}(18)-\mathrm{O}(4)$ & $1.127(15)$ \\
\hline $\mathrm{C}(19)-\mathrm{O}(5)$ & 1.11 & $6(14)$ & $\mathrm{C}(2$ & $(6)$ & $1.150(16)$ & $\mathrm{C}(21)-\mathrm{O}($ & & $1.156(14)$ & $\mathrm{C}(22)-\mathrm{O}(8)$ & $1.141(16)$ \\
\hline $\mathrm{C}(23)-\mathrm{O}(9)$ & 1.14 & $3(16)$ & $\mathrm{C}(2$ & $(10)$ & $1.114(15)$ & $\mathrm{C}(25)-\mathrm{O}($ & 11) & $1.113(15)$ & $\mathrm{C}(26)-\mathrm{O}(12$ & $1.115(15)$ \\
\hline$C(3)-\operatorname{Re}(1)-C$ & & 174.6 & & $\operatorname{Re}(1)$ & $\mathrm{C}(3)-\mathrm{N}(3)$ & $177.5(9)$ & & $\operatorname{Re}(1)-C$ & $(4)-\mathrm{N}(4)$ & $174.2(12)$ \\
\hline $\mathrm{C}(3)-\mathrm{N}(3)-\mathrm{R}$ & & 168.2 & & $\mathrm{C}(21)$ & $\mathrm{Re}(2)-\mathrm{N}(3)$ & $177.6(4)$ & & $C(26)-R$ & $e(3)-N(4)$ & $177.2(5)$ \\
\hline$N(1)-\operatorname{Re}(1)-\Gamma$ & & 74.1 & & $\mathrm{O}(1)$ & $C(1)-\operatorname{Re}(1)$ & $177.9(12)$ & & $\mathrm{O}(2)-\mathrm{C}($ & 2)- $\operatorname{Re}(1)$ & $176.5(11)$ \\
\hline $\mathrm{O}(3)-\mathrm{C}(17)-\mathrm{H}$ & (2) & 178.3 & & $\mathrm{O}(4)$ & $C(18)-\operatorname{Re}(2)$ & $178.0(10)$ & & $\mathrm{O}(5)-\mathrm{C}($ & 19)- $\operatorname{Re}(2)$ & $176.4(10)$ \\
\hline $\mathrm{O}(6)-\mathrm{C}(20)-\mathrm{H}$ & (2) & 175.6 & & $C(4)$ & $\mathrm{N}(4)-\operatorname{Re}(3)$ & $178.1(11)$ & & $\mathrm{O}(7)-\mathrm{C}($ & 21) $-\operatorname{Re}(2)$ & $178.5(11)$ \\
\hline $\mathrm{O}(8)-\mathrm{C}(22)-\mathrm{H}$ & (3) & 177.7 & & $\mathrm{O}(9)$ & $C(23)-\operatorname{Re}(3)$ & $176.2(12)$ & & $\mathrm{O}(10)-\mathrm{C}$ & $(24)-\operatorname{Re}(3)$ & $178.4(11)$ \\
\hline $\mathrm{O}(11)-\mathrm{C}(25)$ & $\operatorname{Re}(3)$ & 175.8 & & $\mathrm{O}(12$ & $-C(26)-\operatorname{Re}(3)$ & $175.1(12)$ & & & & \\
\hline & & & & & & 6 & & & & \\
\hline $\operatorname{Re}(1)-C(1)$ & 1.91 & $8(6)$ & $\operatorname{Re}$ & (2) & $1.927(7)$ & $\operatorname{Re}(1)-C(3)$ & 1.93 & $2(7)$ & $\operatorname{Re}(2)-C(4)$ & $2.075(6)$ \\
\hline $\operatorname{Re}(2)-C(5)$ & 1.90 & $8(6)$ & $\operatorname{Re}$ & (6) & $1.891(6)$ & $\operatorname{Re}(2)-C(7)$ & 2.09 & $3(5)$ & $\operatorname{Re}(3)-C(8)$ & $1.922(6)$ \\
\hline $\operatorname{Re}(3)-C(9)$ & 1.93 & $2(6)$ & $\operatorname{Re}$ & (10) & $1.930(6)$ & $\operatorname{Re}(1)-\mathrm{N}(1)$ & 2.17 & $1(5)$ & $\mathrm{Re}(1)-\mathrm{N}(2)$ & $2.185(5)$ \\
\hline $\operatorname{Re}(1)-\mathrm{N}(3)$ & 2.11 & $9(5)$ & $\operatorname{Re}$ & $\mathrm{J}(4)$ & $2.179(5)$ & $\operatorname{Re}(2)-\mathrm{N}(5)$ & 2.17 & $8(5)$ & $\mathrm{Re}(3)-\mathrm{N}(6)$ & $2.128(5)$ \\
\hline $\operatorname{Re}(3)-\mathrm{N}(7)$ & 2.17 & $5(5)$ & $\operatorname{Re}$ & $\mathrm{J}(8)$ & $2.184(5)$ & $\mathrm{C}(4)-\mathrm{N}(3)$ & 1.15 & $9(8)$ & $\mathrm{C}(7)-\mathrm{N}(6)$ & $1.153(8)$ \\
\hline $\mathrm{C}(1)-\mathrm{O}(1)$ & 1.15 & $4(8)$ & $\mathrm{C}(2$ & & $1.151(8)$ & $\mathrm{C}(3)-\mathrm{O}(3)$ & 1.14 & $0(8)$ & $\mathrm{C}(5)-\mathrm{O}(4)$ & $1.150(8)$ \\
\hline $\mathrm{C}(6)-\mathrm{O}(5)$ & 1.16 & $6(8)$ & $\mathrm{C}(\varepsilon$ & & $1.145(8)$ & $\mathrm{C}(9)-\mathrm{O}(7)$ & 1.14 & $7(8)$ & $\mathrm{C}(10)-\mathrm{O}(8)$ & $1.149(7)$ \\
\hline$C(1)-\operatorname{Re}(1)-\Gamma$ & & 174. & & $\operatorname{Re}(1)$ & $\mathrm{N}(3)-\mathrm{C}(4)$ & $165.6(4)$ & & $\operatorname{Re}(1)-C$ & $(1)-\mathrm{O}(1)$ & 179.2(6) \\
\hline$C(4)-\operatorname{Re}(2)-C$ & & 171. & & $\operatorname{Re}(2)$ & $\mathrm{C}(4)-\mathrm{N}(3)$ & $176.8(5)$ & & $\operatorname{Re}(2)-C$ & $(7)-\mathrm{N}(6)$ & $173.8(5)$ \\
\hline$C(10)-\operatorname{Re}(3)-$ & $\mathrm{J}(6)$ & 175. & & $\operatorname{Re}(3)$ & $\mathrm{N}(6)-\mathrm{C}(7)$ & $169.3(4)$ & & $\operatorname{Re}(3)-C$ & $(10)-O(8)$ & $177.9(5)$ \\
\hline $\operatorname{Re}(1)-C(3)-C$ & & 177.5 & & $\operatorname{Re}(1)$ & $\mathrm{C}(2)-\mathrm{O}(2)$ & $177.0(6)$ & & $\mathrm{N}(1)-\mathrm{Re}$ & $(1)-\mathrm{N}(2)$ & $76.3(19)$ \\
\hline $\operatorname{Re}(2)-C(5)-C$ & & 178.0 & & $\operatorname{Re}(2)$ & $\mathrm{C}(6)-\mathrm{O}(5)$ & $177.4(5)$ & & $\mathrm{N}(4)-\mathrm{Re}$ & $(2)-N(5)$ & $75.6(18)$ \\
\hline $\operatorname{Re}(3)-C(8)-C$ & & 178.1 & & $\operatorname{Re}(3)$ & $\mathrm{C}(9)-\mathrm{O}(7)$ & $178.2(5)$ & & $\mathrm{N}(7)-\mathrm{Re}$ & $(3)-N(8)$ & $75.6(19)$ \\
\hline
\end{tabular}


Table S3. Calculated energies and the compositions of the frontier orbitals of complexes 2, 5 and $\mathbf{6}$.

\begin{tabular}{|c|c|c|c|c|c|c|c|c|c|c|c|c|c|c|c|}
\hline \multirow[t]{3}{*}{2} & \multirow{3}{*}{$\begin{array}{l}\text { Energy } \\
\text { /eV }\end{array}$} & \multicolumn{13}{|c|}{ Contribution to $\mathrm{MO}^{[\mathrm{a}]} / \%$} & \multirow[t]{3}{*}{ Orbital Character } \\
\hline & & \multicolumn{3}{|c|}{ Central Unit } & \multicolumn{5}{|c|}{ Peripheral Unit (1) } & \multicolumn{5}{|c|}{ Peripheral Unit (2) } & \\
\hline & & $\mathrm{d}(\mathrm{Re})$ & $\begin{array}{l}\pi / \pi^{*} \\
(\mathrm{CO})\end{array}$ & $\begin{array}{c}\pi / \pi^{*} \\
\text { (phen) }\end{array}$ & $\mathrm{d}(\mathrm{Re})$ & $\begin{array}{l}\pi / \pi^{*} \\
(\mathrm{CO})\end{array}$ & $\begin{array}{c}\pi / \pi^{*} \\
\text { (phen) }\end{array}$ & $\begin{array}{l}\pi / \pi^{*} \\
(\mathrm{CN})\end{array}$ & $\begin{array}{c}\pi / \pi^{*} \\
\left(\mathrm{PPh}_{3}\right)\end{array}$ & $\mathrm{d}(\mathrm{Re})$ & $\begin{array}{l}\pi / \pi^{*} \\
(\mathrm{CO})\end{array}$ & $\begin{array}{c}\pi / \pi^{*} \\
\text { (phen) }\end{array}$ & $\begin{array}{l}\pi / \pi^{*} \\
(\mathrm{CN})\end{array}$ & $\begin{array}{c}\pi / \pi^{*} \\
\left(\mathrm{PPh}_{3}\right)\end{array}$ & \\
\hline $\mathrm{LUMO}+4$ & -2.511 & & & & & & 6 & & & 1 & & 88 & & 2 & $\pi^{*}($ phen $)$, peripheral \\
\hline $\mathrm{LUMO}+3$ & -2.513 & & & & 1 & & 87 & & 1 & & & 5 & & & $\pi^{*}($ phen $)$, peripheral \\
\hline $\mathrm{LUMO}+2$ & -2.599 & & & 91 & & & & & & & & & & & $\pi^{*}($ phen $)$, central \\
\hline $\mathrm{LUMO}+1$ & -2.711 & & & & & & 9 & & & & & 82 & & 3 & $\pi^{*}($ phen $)$, peripheral \\
\hline LUMO & -2.713 & & & & & & 82 & & 3 & & & 9 & & & $\pi^{*}($ phen $)$, peripheral \\
\hline HOMO & -5.440 & 39 & 10 & & 12 & 3 & & 6 & & 12 & 3 & & 6 & & $\mathrm{~d} \pi(\operatorname{Re})-\mathrm{p} \pi(\mathrm{phen})$, central \\
\hline HOMO-1 & -5.562 & 35 & 10 & & 13 & 4 & & 5 & & 14 & 4 & & 5 & & $\mathrm{~d} \pi(\operatorname{Re})-\mathrm{p} \pi(\mathrm{phen})$, central \\
\hline HOMO-2 & -6.013 & 2 & & & 31 & 8 & & 2 & & 31 & 8 & & 2 & & $\mathrm{~d} \pi(\operatorname{Re})-\mathrm{p} \pi($ phen$)$, peripheral \\
\hline HOMO-3 & -6.055 & 1 & & & 31 & 8 & 2 & 1 & & 30 & 8 & 1 & 1 & & $\mathrm{~d} \pi(\mathrm{Re})-\mathrm{p} \pi(\mathrm{phen})$, peripheral \\
\hline HOMO-4 & -6.349 & 25 & 5 & & 20 & 5 & & 1 & & 21 & 5 & & 1 & & $\mathrm{~d} \pi(\mathrm{Re})-\mathrm{p} \pi(\mathrm{phen})$, peripheral \\
\hline \multirow[t]{3}{*}{5} & \multirow{3}{*}{$\begin{array}{l}\text { Energy } \\
\text { /eV }\end{array}$} & \multicolumn{13}{|c|}{ Atomic Contribution to $\mathrm{MO}^{[\mathrm{a}]} / \%$} & \multirow[t]{3}{*}{ Orbital Character } \\
\hline & & \multicolumn{3}{|c|}{ Central Unit } & \multicolumn{5}{|c|}{ Peripheral Unit (1) } & \multicolumn{5}{|c|}{ Peripheral Unit (2) } & \\
\hline & & $\mathrm{d}(\mathrm{Re})$ & $\begin{array}{l}\pi / \pi^{*} \\
(\mathrm{CO})\end{array}$ & $\begin{array}{c}\pi / \pi^{*} \\
\text { (phen) }\end{array}$ & $\mathrm{d}(\mathrm{R}$ & & $\begin{array}{l}\pi / \pi^{*} \\
(\mathrm{CO}) \\
\end{array}$ & $\begin{array}{c}\pi / \pi^{*} \\
(\mathrm{phen})\end{array}$ & $\begin{array}{l}\pi / \pi^{*} \\
(\mathrm{CN})\end{array}$ & $\mathrm{d}(\mathrm{Re})$ & & & $\begin{array}{c}\pi / \pi^{*} \\
\text { (phen) }\end{array}$ & $\begin{array}{l}\pi / \pi^{*} \\
(\mathrm{CN})\end{array}$ & \\
\hline $\mathrm{LUMO}+4$ & -2.669 & & & & 6 & & 36 & & & 6 & 4 & & & & $\pi *(\mathrm{CO})$, peripheral \\
\hline $\mathrm{LUMO}+3$ & -2.670 & & & 98 & & & & & & & & & & & $\pi^{*}(\mathrm{phen})$, central \\
\hline $\mathrm{LUMO}+2$ & -2.700 & 2 & & 64 & 2 & & 8 & & 1 & 2 & rata & & & 1 & $\pi^{*}(\mathrm{phen})$, central \\
\hline LUMO+1 & -2.899 & & & 26 & 6 & & 28 & & & 6 & & & & & $\begin{array}{l}\pi^{*}(\mathrm{CO}) \text {, peripheral }+\pi^{*}(\text { phen }), \\
\text { central }\end{array}$ \\
\hline LUMO & -2.900 & 1 & & & 6 & & 44 & & 3 & 6 & 4 & & & 3 & $\pi^{*}(\mathrm{CO})$, peripheral \\
\hline HOMO & -5.820 & 61 & 14 & & 1 & & & & 7 & 1 & & & & 7 & $\mathrm{~d} \pi(\mathrm{Re})-\mathrm{p} \pi(\mathrm{phen})$, central \\
\hline HOMO-1 & -5.962 & 56 & 14 & 8 & 1 & & & & 7 & 1 & & & & 7 & $\mathrm{~d} \pi(\mathrm{Re})-\mathrm{p} \pi(\mathrm{phen})$, central \\
\hline HOMO-2 & -6.584 & 67 & 30 & 4 & & & & & & & & & & & $\mathrm{~d} \pi(\mathrm{Re})-\mathrm{p} \pi(\mathrm{phen})$, central \\
\hline HOMO-3 & -6.798 & & & 94 & & & & & & & & & & & $\pi($ phen $)$, central \\
\hline HOMO-4 & -6.849 & & & 94 & & & & & & & & & & & $\pi($ phen$)$, central \\
\hline
\end{tabular}




\begin{tabular}{|c|c|c|c|c|c|c|c|c|c|c|c|c|c|}
\hline \multirow[t]{3}{*}{6} & \multirow{3}{*}{$\begin{array}{c}\text { Energy } \\
\text { /eV }\end{array}$} & \multicolumn{11}{|c|}{ Contribution to $\mathrm{MO}^{[\mathrm{a}]} / \%$} & \multirow[t]{3}{*}{ Orbital Character } \\
\hline & & \multicolumn{3}{|c|}{ Central Unit } & \multicolumn{4}{|c|}{ Peripheral Unit (1) } & \multicolumn{4}{|c|}{ Peripheral Unit (2) } & \\
\hline & & $\mathrm{d}(\mathrm{Re})$ & $\begin{array}{l}\pi / \pi^{*} \\
(\mathrm{CO})\end{array}$ & $\begin{array}{c}\pi / \pi^{*} \\
\text { (phen) }\end{array}$ & $\mathrm{d}(\mathrm{Re})$ & $\begin{array}{l}\pi / \pi^{*} \\
(\mathrm{CO})\end{array}$ & $\begin{array}{c}\pi / \pi^{*} \\
\text { (phen) }\end{array}$ & $\begin{array}{l}\pi / \pi^{*} \\
(\mathrm{CN}) \\
\end{array}$ & $\mathrm{d}(\mathrm{Re})$ & $\begin{array}{l}\pi / \pi^{*} \\
(\mathrm{CO})\end{array}$ & $\begin{array}{c}\pi / \pi^{*} \\
\text { (phen) }\end{array}$ & $\begin{array}{l}\pi / \pi^{*} \\
(\mathrm{CN}) \\
\end{array}$ & \\
\hline $\mathrm{LUMO}+4$ & -2.479 & & & & & & 45 & & & & 49 & & $\pi^{*}$ (phen), peripheral \\
\hline $\mathrm{LUMO}+3$ & -2.659 & & & & & & 49 & & & & 46 & & $\pi^{*}($ phen $)$, peripheral \\
\hline $\mathrm{LUMO}+2$ & -2.815 & & & 92 & & & & & & & & & $\pi^{*}($ phen $)$, central \\
\hline $\mathrm{LUMO}+1$ & -2.879 & & & & & & 44 & & & & 44 & & $\pi^{*}($ phen $)$, peripheral \\
\hline LUMO & -2.884 & & & & & & 44 & & & & 45 & & $\pi^{*}($ phen $)$, peripheral \\
\hline HOMO & -6.099 & 54 & 14 & & 4 & & & 7 & 4 & & & 7 & $\mathrm{~d} \pi(\mathrm{Re})-\mathrm{p} \pi(\mathrm{phen})$, central \\
\hline HOMO-1 & -6.226 & 52 & 12 & 4 & 4 & & & 7 & 4 & & & 7 & $\mathrm{~d} \pi(\operatorname{Re})-\mathrm{p} \pi(\mathrm{phen})$, central \\
\hline HOMO-2 & -6.733 & 67 & 30 & 2 & & & & & & & & & $\mathrm{~d} \pi(\operatorname{Re})-\mathrm{p} \pi(\mathrm{phen})$, central \\
\hline HOMO-3 & -7.447 & 2 & & & 29 & 11 & & 3 & 29 & 11 & & 3 & $\mathrm{~d} \pi(\mathrm{Re})-\mathrm{p} \pi($ phen$)$, peripheral \\
\hline HOMO-4 & -7.810 & & & & 27 & 12 & 3 & 1 & 27 & 12 & 3 & 1 & $\mathrm{~d} \pi(\mathrm{Re})-\mathrm{p} \pi($ phen$)$, peripheral \\
\hline
\end{tabular}

${ }^{[a]}$ Due to rounding, the total contributions may not equal to $100 \%$ 


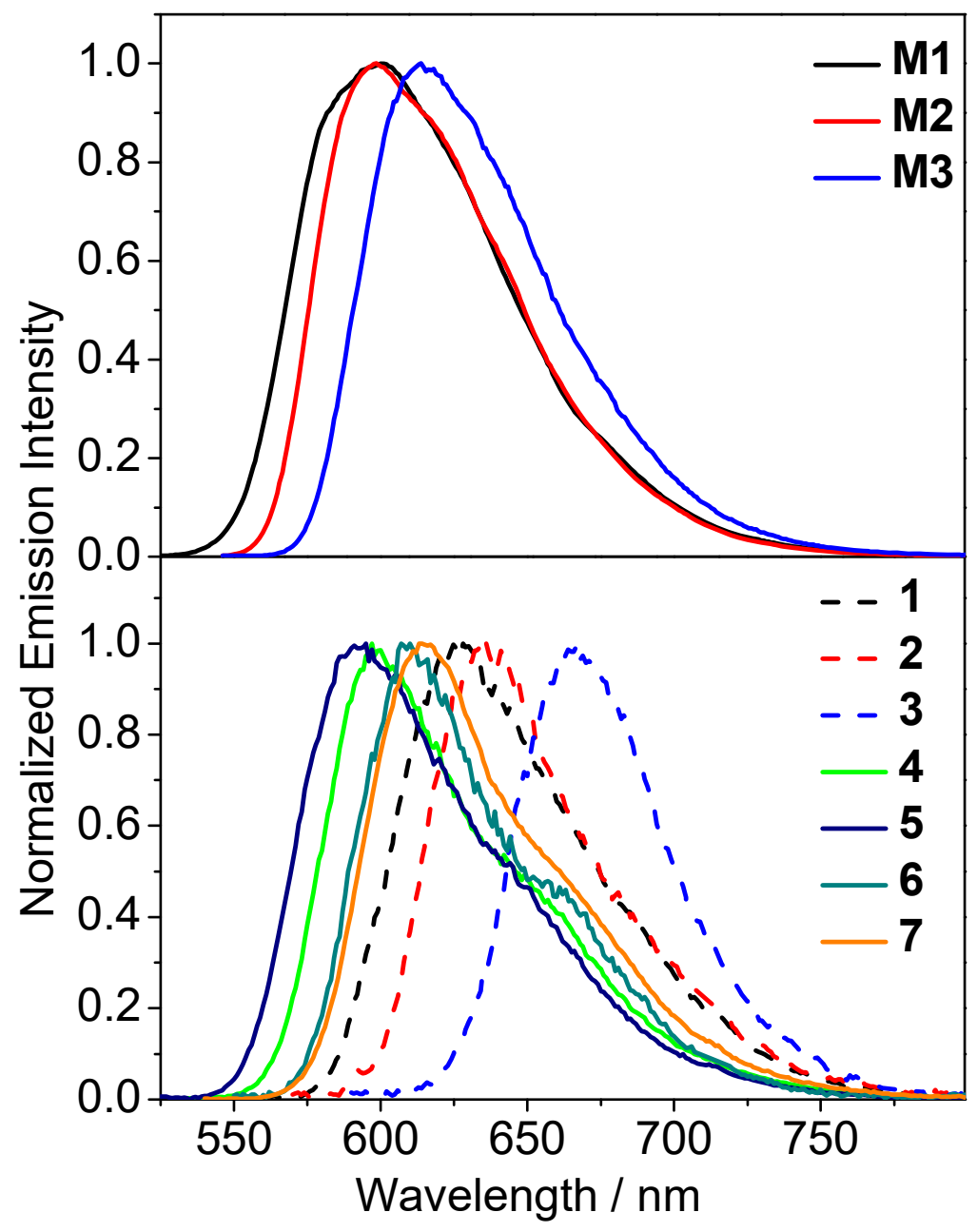

Figure S1. Overlaid emission spectra of M1-M3 (top) and 1-7 (bottom) in EtOH/MeOH (4:1) glass at $77 \mathrm{~K}$. 


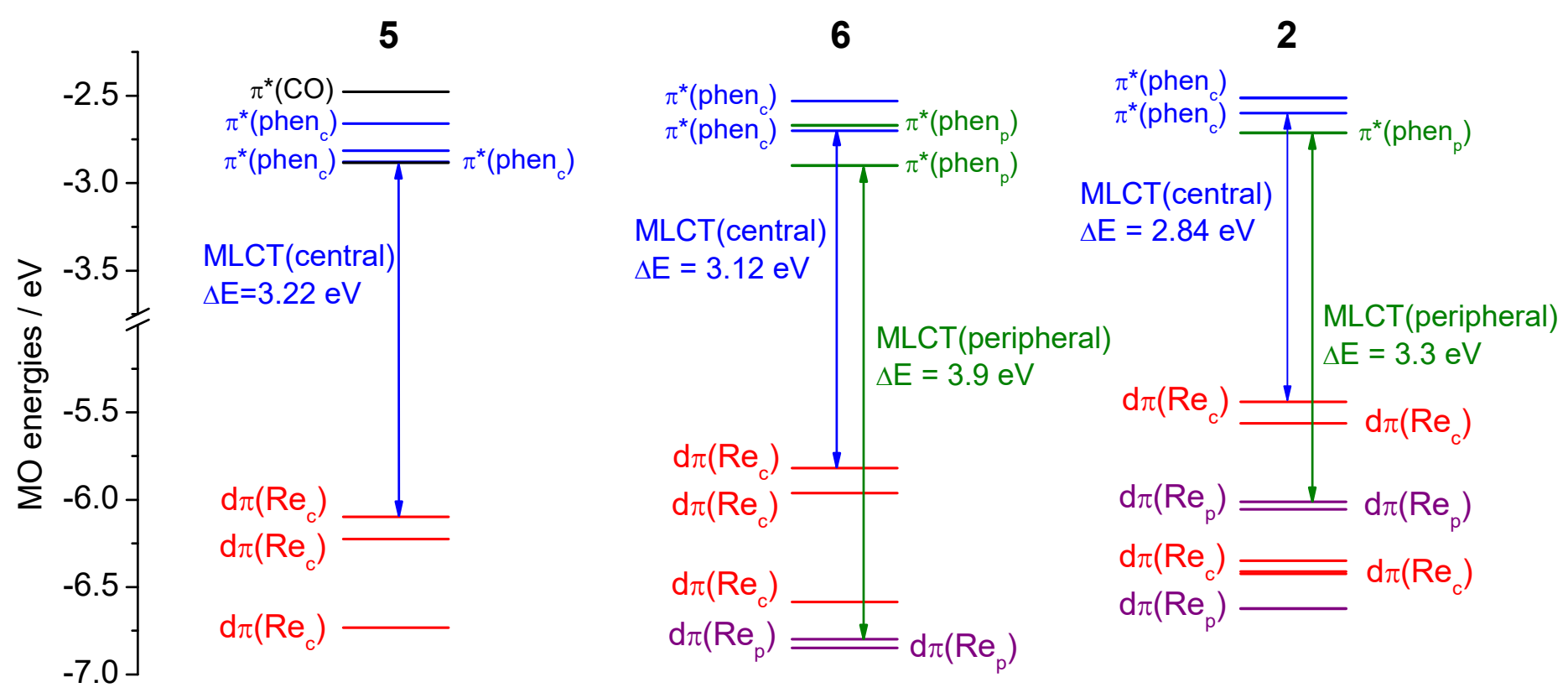

Figure S2. Schematic diagram for the calculated orbital energy levels of 2, 5 and $\mathbf{6}$. $\operatorname{Re}_{\mathrm{c}}$ and $\mathrm{Re}_{\mathrm{p}}$ are rhenium metal centers of the central and peripheral coordination units, respectively; phen $_{c}$ and $\operatorname{phen}_{\mathrm{p}}$ are the phenanthroline ligands of the central and peripheral coordination units, respectively. 


\section{Reference}

1. Demas, J. N.; Crosby, G. A. Measurement of photoluminescence quantum yields. J. Phys. Chem. 1971, 75, 991-1024.

2. Suzuki, K.; Kobayashi, A.; Kaneko, S.; Takehira, K.; Yoshihara, T.; Ishida, H.; Shiina, Y.; Oishic, S.; Tobita, S. Reevaluation of absolute luminescence quantum yields of standard solutions using a spectrometer with an integrating sphere and a back-thinned CCD detectorPhys. Chem. Chem. Phys. 2009, 11, 9850-9860.

3. SHELX-97, Sheldrick, G. M. SHELX-97: Programs for Crystal Structure Analysis (Release 97-2). University of Göttingen, Germany, 1997.

4. Frisch, M. J.; Trucks, G.W.; Schlegel, H. B.; et al. Gaussian 09, Revision B.01; Gaussian, Inc.: Wallingford, CT, 2009.

5. (a) Lee, C.; Yang, W.; Parr, R. G. Development of the Colle-Salvetti correlation-energy formula into a functional of the electron density. Phys. Rev. B: Condens. Matter Mater. Phys. 1988, 37, 785-789. (b) Becke, A. D. Density-functional thermochemistry. III. The role of exact exchange. J. Chem. Phys. 1993, 98, 5648-5652.

6. (a) Hay, P. J.; Wadt, W. R. Ab initio effective core potentials for molecular calculations. Potentials for K to Au including the outermost core orbitals. J. Chem. Phys. 1985, 82, 299-310. (b) Hay, P. J.; Wadt, W. R. Ab initio effective core potentials for molecular calculations. Potentials for the transition metal atoms Sc to Hg. J. Chem. Phys. 1985, 82, 270-283.

7. Scalmani, G.; Frisch, M. J. Continuous surface charge polarizable continuum models of solvation. I. General formalism. J. Chem. Phys. 2010, 132, 114110. 